\title{
Zonisamide in children and young adults with refractory epilepsy: An open label, multicenter Italian study
}

\author{
Giangennaro Coppola ${ }^{a, *}$, Salvatore Grosso ${ }^{d}$, Alberto Verrotti ${ }^{f}$, \\ Pasquale Parisi ${ }^{c}$, Anna Luchetti ${ }^{c}$, Emilio Franzoni ${ }^{g}$, Salvatore Mangano ${ }^{\text {h, }}$ \\ Andrea Pellicciac, Francesca Felicia Operto ${ }^{a}$, Paola lannetti ${ }^{\mathrm{e}}$, \\ Paolo Curatolo ${ }^{b}$, Paolo Balestri ${ }^{d}$, Antonio Pascotto ${ }^{a}$
}

\footnotetext{
a Clinic of Child Neuropsychiatry, Second University of Naples, Via Pansini 5, 84131 Naples, Italy

b Department of Neuroscience, Division of Pediatric Neurology, Tor Vergata University of Rome, Italy

c Child Neurology \& Paediatric Sleep Centre, La Sapienza University II of Rome, Italy

d Pediatric Neurology Section, Department of Pediatrics, University of Siena, Siena, Italy

e Division of Child Neurology, Department of Paediatrics, La Sapienza University II of Rome, Italy

f Section of Pediatrics, Department of Pediatrics, University of Chieti, Italy

g Clinic of Neuropediatrics, Department of Pediatrics, University of Bologna, Italy

h Clinic of Child Neuropsychiatry, Department of Pediatrics, University of Palermo, Italy
}

Received 1 February 2008; received in revised form 5 October 2008; accepted 17 October 2008 Available online 10 December 2008

\section{KEYWORDS \\ Epilepsy; \\ Zonisamide; \\ Pediatric epilepsy; \\ Antiepileptic drugs}

\begin{abstract}
Summary
Purpose: To report on the first multicenter Italian experience with zonisamide as an add-on drug for refractory generalised or partial epilepsy in children, adolescents and young adults. Methods: The patients were enrolled in a prospective, add-on, open-label treatment study from eight Italian centres for children and adolescent epilepsy care. Eighty-two young patients (45 males, 37 females), aged between 3 and 34 years (mean 13.1 years), all affected by partial (47) or generalised (35) refractory epilepsy, were enrolled in the study.

ZNS was added to the baseline therapy at a starting dose of $1 \mathrm{mg} / \mathrm{kg} /$ day twice daily. This dose was increased by $2 \mathrm{mg} / \mathrm{kg}$ every $1-2$ weeks over a period of up 3 months, according to the patient's response and tolerability, up to a maximum dose of $12 \mathrm{mg} / \mathrm{kg}$. ZNS was given at the mean daily dose of $5.7 / \mathrm{mg} / \mathrm{kg} / 24 \mathrm{~h}$ (range $1-12 \mathrm{mg} / \mathrm{kg}$ ).

Results: After a mean follow-up period of 11.9 months (range 2-64 months), 9 patients (10.9\%) were seizure-free. The number of seizures decreased by $50-99 \%$ in 31 cases (37.8\%), by $25-49 \%$ in 5 cases $(6.1 \%)$, remained the same in 29 cases $(35.4 \%)$ and increased in 8 cases $(9.7 \%)$.
\end{abstract}

\footnotetext{
* Corresponding author. Tel.: +39 081 5666695; fax: +39 0815666694 .

E-mail address: giangennaro.coppola@unina2.it (G. Coppola).
} 
After 15 months of follow-up, 61 patients (74.4\%) were still taking ZNS, while the remaining 21 (25.6\%) had stopped. Twenty-two patients $(26.8 \%)$ reported adverse effects while taking ZNS. They generally appeared during the first weeks of treatment, and were mild to moderate. The most frequent adverse effects were irritability and a reduced appetite.

Conclusion: ZNS effectively reduced seizure frequency in this pediatric population with both partial and generalised crypto/symptomatic refractory epilepsy. Its overall tolerability was good. (c) 2008 Elsevier B.V. All rights reserved.

\section{Introduction}

The novel antiepileptic drug zonisamide (ZNS) is characterised by a broad range of pharmacological effects associated with multiple mechanisms of action. These include a voltage-sensitive sodium and T-type calcium channel blockade, a reduction in glutamate-mediated synaptic excitation and enhanced inhibitory effects mediated by gamma-aminobutyrric acid (Biton, 2004).

Zonisamide has recently been approved in Europe for the adjunctive treatment of refractory partial seizures in adults. It has been available in Japan since, 1989, with studies there having established that ZNS is effective and safe for use in children (Ohtahara, 2006). Information from other countries concerning its use in pediatric patients is still somewhat limited. There are, however, a growing number of studies reporting the clinical efficacy of ZNS, both as add-on therapy (Kim et al., 2005; Mandelbaum et al., 2005; Santos and Brotherton, 2005; Ohtahara, 2006; Kluger et al., 2008) and as monotherapy (Miura, 2004; Seki et al., 2004; Kim et al., 2005; Kothare et al., 2006) in pediatric generalised and partial seizures.

The aim of this paper is to report on the first multicentre Italian experience with ZNS as an add-on drug for the treatment of refractory generalised and partial epilepsy in children, adolescents and young adults.

\section{Methods}

Patients were recruited in a prospective add-on, open-label treatment study from eight Italian centres for pediatric and adolescent epilepsy care. The patients were selected according to the following criteria: (1) age one year and over; (2) partial or generalised epileptic seizures refractory to at least three previous antiepileptic drugs (AEDs), alone or in combination; (3) more than 1 seizure per month in the last 6 months; (4) use of at least one other AED, but no more than two, at baseline; (5) informed consent from parents/caregivers, who had to be able to administer the study drug and record seizures in a diary. Moreover, female patients of childbearing age were required not to be pregnant and to be using an adequate form of contraception.

Exclusion criteria included progressive neurological or systemic disease. Patients with significantly abnormal liver, kidney and blood laboratory values were also excluded, as were those who were considered to be unlikely to comply with the study requirements.

Besides enrolling ZNS-naïve subjects, study sites that had previously started patients on ZNS had the option of enrolling patients who had completed at least 6 months of maintenance treatment and met the study inclusion and exclusion criteria.

Pseudo-seizures were excluded by means of video-EEG recordings and/or long-term monitoring EEGs. CT and MRI were performed in all cases. The number of seizures was recorded by parents and caregivers at home and at school. Seizure frequency, type and duration were recorded in an epilepsy diary to be kept and reviewed at each follow-up visit. Seizures were classified according to the International League against Epilepsy (ILAE) classification of epileptic seizures (ILAE Commission, 1981), while severe myoclonic epilepsy in infancy was diagnosed according to Dravet's criteria (Dravet et al., 1984).

There was an initial observation period of 6 months (baseline), during which antiepileptic treatment was generally left unchanged unless particular reasons arose, such as the occurrence of status epilepticus. If seizures occurred daily, the observation period could be shortened to 3 months.

After the observation period, ZNS was added to the baseline therapy at the starting dose of $1 \mathrm{mg} / \mathrm{kg} /$ day twice daily. The dose was increased by $2 \mathrm{mg} / \mathrm{kg}$ every $1-2$ weeks over a period of up to 3 months. The dose was adjusted at the investigator's discretion according to the patient's response and tolerability, up to a maximum dose of $12 \mathrm{mg} / \mathrm{kg}$. Patients procured zonisamide on their own during the trial period. Concomitant therapy was generally not modified at any time during the study. If any adverse event occurred, the titration phase could be extended according to the patient's clinical conditions. Rescue drugs were allowed whenever necessary. EEG, adverse effects and blood levels of concomitant AEDs were monitored in all patients. Patients were followed on a weekly basis during the titration period, either by means of visits to the clinic or by telephone. Patients subsequently visited the clinic at 3month intervals during the maintenance treatment, with a monthly follow-up by telephone between visits to the clinic whenever necessary. Blood chemistry and liver and kidney function were carefully assessed at each time interval. Parents/caregivers were informed of the potential clinical adverse effects to refer to the clinician.

The response to treatment, in relation to the baseline, was rated as follows: (i) seizure control (100\% seizure remission); (ii) $50-99 \%$ decrease in number of seizures; (iii) $25-49 \%$ decrease in number of seizures; (iv) unchanged when the seizure rate remained the same; (v) worsened when the seizure rate increased.

The Institutional Review Board from each epilepsy unit approved the study; no support was received from pharmaceutical companies.

The statistical analysis was performed by means of a two-tailed Wilcoxon Rank test for non-parametric data and the $\chi^{2}$ test.

\section{Results}

Eighty-two young patients (45 males, 37 females), aged between 3 and 34 years (<6 years: 20.7\%; 7-12 years: $35.4 \%$; $13-18$ years: $23.2 \%$; $>19$ years: $20.7 \%$ : mean age 13.1 years), all affected by refractory epilepsy, were enrolled in the study. Patient diagnoses were as follows: symptomatic (28) and cryptogenic (19) partial epilepsy; symptomatic (19) and cryptogenic (16) generalized epilepsy (including 2 with Lennox-Gastaut syndrome, 3 with myoclonic-astatic syndrome, 6 with severe myoclonic epilepsy in infancy, and 2 with continuous spike and wave status during slow sleep. The mean duration of epilepsy was 10.2 years (range $1-31$ years). Seizure types, which were often combined, were as follows: complex partial (24), secondarily generalised partial seizures (21), 
Table 1 Response to treatment with zonisamide according to epilepsy type.

\begin{tabular}{|c|c|c|c|c|c|c|c|c|}
\hline \multirow[t]{3}{*}{ Seizure decrease (\%) } & \multicolumn{4}{|c|}{ Partial epilepsy $(n=47)^{n}$} & \multicolumn{4}{|c|}{ Generalised epilepsy $(n=35)^{n}$} \\
\hline & \multicolumn{2}{|c|}{ Cryptogenic } & \multicolumn{2}{|c|}{ Symptomatic } & \multicolumn{2}{|c|}{ Cryptogenic } & \multicolumn{2}{|c|}{ Symptomatic } \\
\hline & $N$ & $\%$ & $N$ & $\%$ & $N$ & $\%$ & $N$ & $\%$ \\
\hline 100 & $7^{n}$ & 36.8 & $0^{n}$ & & $2^{n}$ & 12.5 & $0^{n}$ & \\
\hline 50-99 & $7^{n}$ & 36.8 & $13^{n}$ & 46.4 & $5^{n}$ & 31.2 & $6^{n}$ & 31.6 \\
\hline $25-49$ & 0 & & 1 & 3.6 & 2 & 12.5 & 2 & 10.5 \\
\hline Unchanged & 3 & 15.8 & 11 & 39.3 & 7 & 43.8 & 8 & 42.1 \\
\hline Increased & 2 & 10.5 & 3 & 10.7 & 0 & & 3 & 15.8 \\
\hline Total & 19 & 100.0 & 28 & 100.0 & 16 & 100.0 & 19 & 100.0 \\
\hline
\end{tabular}

tonic (19), epileptic spasms (10), tonic-clonic (10), atonic (8), myoclonic (7).

Seizure frequency at baseline was: $\geq 1 /$ day in 49 patients, $5-15 /$ month in 19 patients, and $1-4 /$ month in 14 patients. The mean total number of seizures per month at baseline was 97.0 (range 4-900).

CT/MRI imaging scans disclosed abnormal findings in 47 patients (57.3\%): brain atrophy (16), gliosis (14), migration disorders (12), tuberous sclerosis (2), poroencephaly (1), tetraventricular hydrocephalus (1), dysembrioplastic neuroepithelioma (1). This last case was included because serial MRI scans ruled out growth of the tumor.

Developmental psychomotor and mental level was normal in 14 patients (17\%); a mild delay was found in $14(17 \%)$, a moderate delay in $9(10.9 \%)$, and severe/profound delay in 45 patients $(54.9 \%)$.

The neurological examination was abnormal in 39 patients (47.6\%): 21 patients had spastic tetraparesis, 3 generalised hypotonia, 3 diplegia, 4 hemiparesis, 2 double hemiparesis, 1 hypotonic syndrome and 5 ataxia.

Five patients were taking one other AED concomitantly (PB, OXC, VGB, CBZ, NZP), 77 were taking two. The following AEDs were taken in various combinations with ZNS: VPA (32), BDZ (29), CBZ (17), PB(16), LEV (13), LTG (11), TPM (13), PHT (8), OXC (8), FBM (5), VGB (5), AZM (5), GBP (2), PGB (1), STP (1), KD (1).

ZNS was given at a mean daily dose of $5.7 / \mathrm{mg} / \mathrm{kg} / 24 \mathrm{~h}$ (range $1-12 \mathrm{mg} / \mathrm{kg} /$ day).

\section{Efficacy}

After a mean follow-up period of 11.9 months (range 2-64 months), 9 patients were seizure-free (10.9\%; mean followup 11.2 months). A 50-99\% reduction in seizure frequency was found in 31 cases (37.8\%), a $25-49 \%$ in 5 cases $(6.1 \%)$, while seizure frequency remained unchanged in 29 patients $(35.4 \%)$ and increased in 8 patients $(9.7 \%)$ (Table 1$)$.

Overall, ZNS was more effective (though not significantly) in partial than in generalised epilepsy $(57.4 \%$ vs $37.1 \%$, respectively; $p=.377$ ), and, though to a lesser extent, in both partial $(p=.478)$ and generalised $(p=.860)$ cryptogenic epilepsy.

As for the epilepsy type, 4 of the 6 patients with Dravet syndrome did not respond, while the remaining 2 had a $\geq 50 \%$ reduction in the number of tonic-clonic and myoclonic seizures.

Two of the three patients with myoclonic-astatic epilepsy had a $50 \%$ reduction in the number of seizures, while two patients with Lennox-Gastaut syndrome did not improve. Two other children, aged 9 and 11 years, with a continuous spike-and-wave status during slow sleep EEG pattern and monthly seizures, were seizure-free after 6 and 3 months. EEG showed a significant decrease in the spike-andwave index during slow sleep stages in one, while it was unchanged in the other.

As for seizure type, ZNS was most effective (i.e. $\geq 50 \%$ reduction in seizure frequency) in the treatment of partial seizures with or without secondary generalisation, tonic seizures and epileptic spasms (Fig. 1).

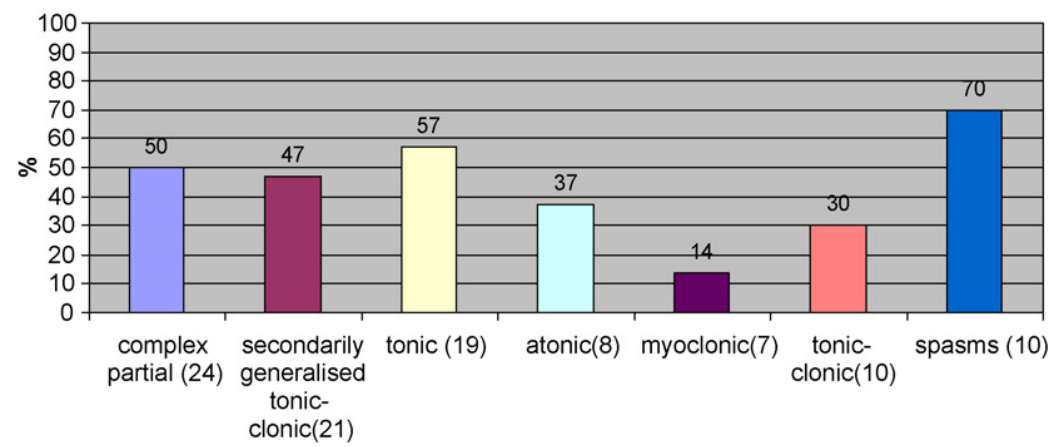

Figure 1 Percentages of patients according to seizure type who experienced a $50 \%$ or greater reduction in seizure frequency when ZNS was added to their baseline therapy. 


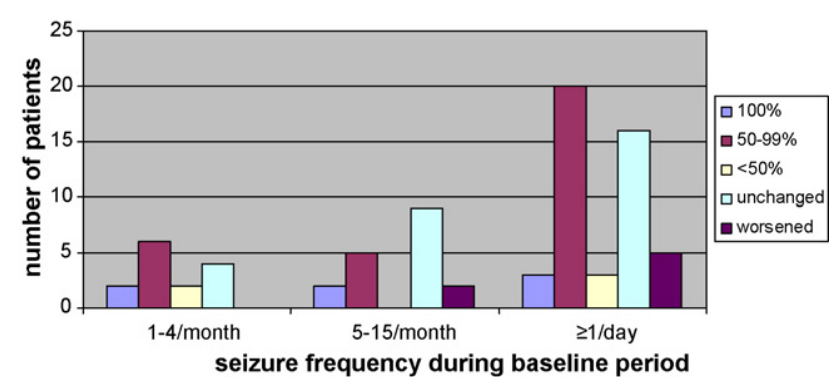

Figure 2 Response to ZNS therapy according to seizure frequency during baseline period. (For interpretation of the references to color in this figure legend, the reader is referred to the web version of the article.)

No significant relationship was found between responders and non-responders in age at exposure to ZNS, duration of epilepsy, aetiology, sex and baseline therapy $(p>0.5$; Wilcoxon Rank Sum test).

Seizure frequency did not increase when ZNS was added to therapy in patients with a lower baseline seizure frequency (Fig. 2).

Seizure frequency increased following addition of ZNS in 8 patients $(9.7 \%)$. Fig. 3 shows that the increase in seizure frequency following the addition of ZNS was comparable in the different seizure types. An increase in seizure frequency generally occurred during the first weeks of treatment at daily doses of $0.5-2 \mathrm{mg} / \mathrm{kg}$. In two patients, seizures became very frequent but promptly stopped after ZNS withdrawal. The mean age in this group of 8 patients was 13.7 years and the seizure frequency was $\geq 1$ /day at baseline in $75 \%$ of patients.

After 15 months of follow-up, 61 pts (74.4\%) were still taking ZNS, while the remaining 21 (25.6\%) had stopped taking the drug owing to its lack of efficacy in reducing seizure frequency, even at the maximum doses used (see Fig. 4, Kaplan-Meyer survival curve).

The mean total number of seizures per month during follow-up was 71.4 (range 0.2-660).

\section{Tolerability}

Twenty-two patients (26.8\%) reported adverse effects while taking ZNS (Table 2). Adverse effects generally appeared during the first weeks of treatment and were mild to moderate in severity. Only one patient stopped treatment because

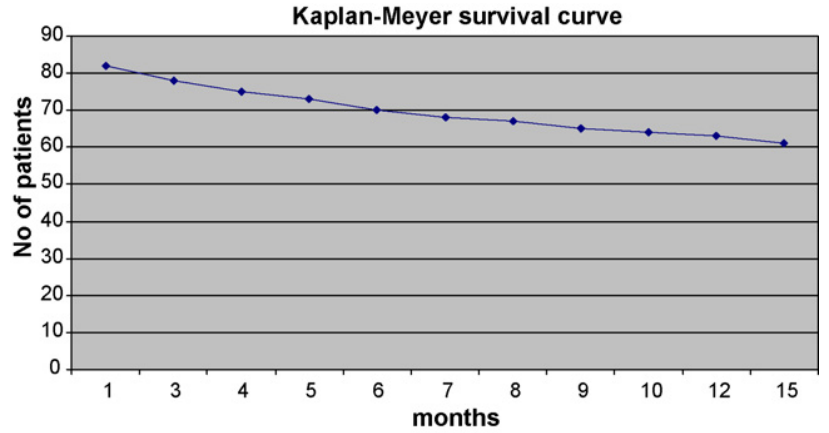

Figure 4 Kaplan-Meyer survival curve for 15-month treatment with ZNS showing the number of patients still being treated.

Table 2 All treatment-emergent adverse effects during the follow-up period.

\begin{tabular}{ll}
\hline & (No. of patients) \\
\hline Body as a whole & Weakness, 5 \\
& Weight gain, 1 \\
& Adenomegaly, 1 \\
& Transient vomiting, 1 \\
Digestive & Decreased appetite, 3 \\
& Abdominal pain, 1 \\
& \\
Nervous system & Irritability, 9 \\
& Drowsiness, 2 \\
Insomnia, 2 & Inattention, 1 \\
& Cephalalgia, 1 \\
& Choreoathetosis, 1 \\
& \\
Skin & Rash, 1 \\
\hline
\end{tabular}

of adverse effects: this patient developed a skin rash ten days after starting ZNS therapy. ZNS was suspended and tried again, but when the skin rash reappeared, the drug was definitively tapered off.

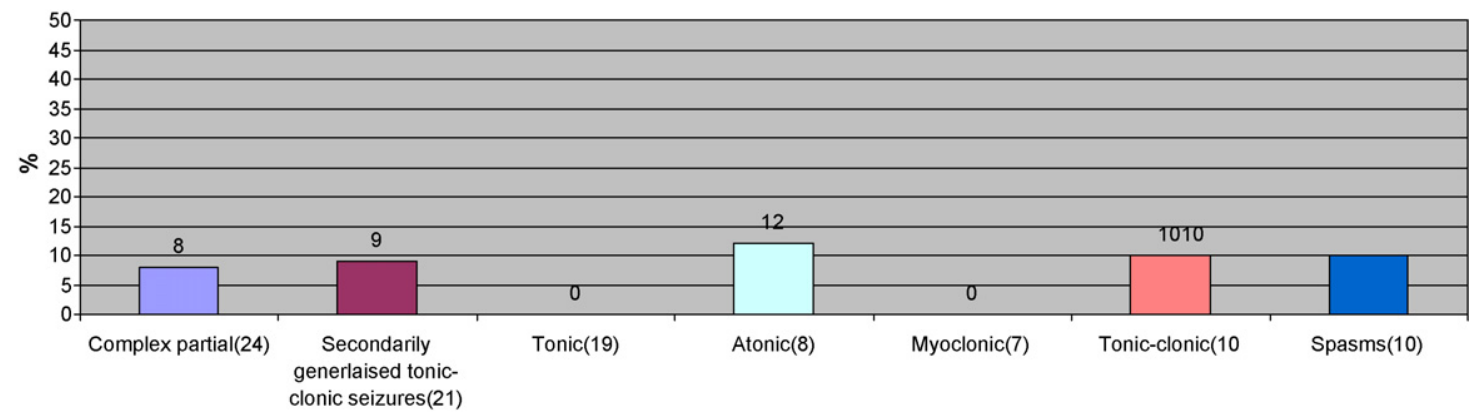

Figure 3 Percentages of patients who experienced a 25\% or greater increase in seizure frequency when ZNS was added to their treatment regimen. 
The most frequent adverse effects, which tended to be CNS-related (e.g. irritability and drowsiness), were generally eliminated by reducing the daily dosage of ZNS or by slowing down the drug titration.

All the patients' laboratory test values were normal.

\section{Discussion}

In this study, ZNS as add-on therapy significantly reduced seizure frequency in approximately half of our patients who had partial or generalised epilepsy that had proved resistant to at least three previous antiepileptic drugs.

Few patients $(10.9 \%)$, all of whom were in the cryptogenic group, became seizure-free. In addition, ZNS proved to be somewhat more effective in the treatment of partial seizures.

The overall response to ZNS observed in our study is in keeping with that reported in Japanese studies and the few small-scale studies conducted to date in Europe and the US on pediatric populations with both partial and generalised treatment-resistant seizures (38-58\% by Kim et al., 2005; Mandelbaum et al., 2005; Santos and Brotherton, 2005; linuma and Haginoya, 2004; Ohtahara, 2006; Kluger et al., 2008). The number of seizure-free patients $(10.9 \%)$ in our series was somewhat lower than that in other studies $(16 \%$ and $25 \%$ by Santos and Brotherton (2005), and by Kim et al. (2005), respectively), though this may be ascribed to the severe refractoriness of our patients.

Worthy of note is the good response to ZNS of drugresistant epileptic spasms, which is in keeping with findings reported by Lotze and Wilfong (2004). Interestingly, ZNS was to some extent effective in focal-onset tonic seizures as well.

The effect of ZNS was poor in most of the children in our series with Dravet syndrome and myoclonic-astatic epilepsy, as well as in the two patients with Lennox-Gastaut syndrome. Although no conclusions can be drawn from this finding owing to the small number of patients with these specific syndromes, it is noteworthy that You et al. (2007) reported $100 \%$ seizure control in $4.8 \%$ and a $>50 \%$ seizure reduction in a further $46.8 \%$ of 62 children with Lennox-Gastaut syndrome treated with ZNS as long-term adjunctive therapy.

The data yielded by our study regarding seizure worsening are in keeping with those by Kim et al. (2005) $(9 \%$ and $14.5 \%$, respectively). An increase in seizure frequency occurred above all in the first weeks of treatment, often during the dose-escalation phase, with no relationship with seizure type being found. If an increase in seizure frequency did occur, values promptly returned to those at baseline after ZNS withdrawal; the explanation for this increase is thus more likely to be ascribable to a direct role of ZNS as opposed to a natural fluctuation in seizure frequency.

The retention rate observed in our series is consistent with that reported by Kluger et al. (2008) (60\% and $41.7 \%$, respectively), though somewhat higher. This high retention rate may be attributed not so much to the efficacy of ZNS as to its overall good tolerability.
Most of the adverse effects, including somnolence, nervousness, and decreased appetite, were related to the central nervous system. On the whole, these adverse effects were mild to moderate, and mostly occurred during titration dosing and resolved in maintenance dosing. No serious side effects were observed.

In conclusion, in our experience ZNS reduced seizure frequency in young patients with partial and generalised crypto/symptomatic refractory epilepsy. Moreover, ZNS was generally well tolerated in this population study. Further studies are warranted to gain a better understanding of the efficacy of ZNS both in the long term and in the treatment of well-defined epileptic syndromes.

\section{References}

Biton, V., 2004. Zonisamide: newer antiepileptic agent with multiple mechanisms of action. Expert Rev. Neurother. 4, 935-943.

Dravet, C., Roger, J., Bureau, M., 1984. L'epilepsie myoclonicque severe du nourisson. In: Roger, J., Dravet, C., Bureau, M., Dreifuss, F.E., Wolf, P. (Eds.), Les syndromes épilepticques de l'enfant et de l'adolescent. John Libbey Eurotext Ltd., pp. 58-67.

linuma, K., Haginoya, K., 2004. Clinical efficacy of zonisamide in childhood epilepsy after long-term treatment: a postmarketing, multi-institutional survey. Seizure 13 (Suppl 1), S34-39.

Commission on classification and terminology of the International League against Epilepsy, 1981. Proposal for revised clinical and electroencephalographic classification of epileptic seizures. Epilepsia 22, 489-501.

Kim, H.L., Aldridge, J., Rho, J.M., 2005. Clinical experience with zonisamide monotherapy and adjunctive therapy in children with epilepsy at a tertiary care referral center. J. Child Neurol. 20 (3), 212-219.

Kluger, G., Zsoter, A., Holthausen, H., 2008. Long-term use of zonisamide in refractory childhood-onset epilepsy. Eur. J. Paediatr. Neurol. 12 (1), 19-23.

Kothare, S.V., Kaleyias, J., Mostofi, N., Valencia, I., Melvin, J.J., Hobdell, E., Khurana, D.S., Legido, A., 2006. Efficacy and safety of zonisamide monotherapy in a cohort of children with epilepsy. Pediatr. Neurol. 34 (5), 351-354.

Lotze, T.E., Wilfong, A.A., 2004. Zonisamide treatment for symptomatic infantile spasms. Neurology 27 (2), 296-298.

Mandelbaum, D.E., Bunch, M., Kugler, S.L., Venkatasubramanian, A., Wollack, J.B., 2005. Broad-spectrum efficacy of zonisamide at 12 months in children with intractable epilepsy. J. Child Neurol. 20 (7), 594-597.

Miura, H., 2004. Zonisamide monotherapy with once-daily dosing in children with cryptogenic localization-related epilepsies: clinical effects and pharmacokinetic studies. Seizure 13 (Suppl 1), S17-23.

Ohtahara, S., 2006. Zonisamide in the management of epilepsyJapanese experience. Epilepsy Res. 68 (Suppl 2), S25-33.

Santos, C.C., Brotherton, T., 2005. Use of zonisamide in pediatric patients. Pediatr. Neurol. 33 (1), 12-14.

Seki, T., Kumagai, N., Maezawa, M., 2004. Effects of zonisamide monotherapy in children with epilepsy. Seizure 13 (Suppl 1), S26-32.

You, S.J., Kang, H.C., Kim, H.D., Lee, H.S., Ko, T.S., 2007. Clinical efficacy of zonisamide in Lennox-Gastaut syndrome: Korean multicentric experience. Brain Dev. 22 [Epub ahead of print]. 